\title{
Verwischte Gesichter. Grenzen der Interpretation automatisch erzeugter Bilder
}

\author{
Felix Keller
}

Automatisch erstellte Bilder beleben zusehends die gesellschaftliche Wirklichkeit: visuelle Formen, Lebensformen vielleicht, die entstehen, wenn technische Installationen autark mit unterschiedlichen Realitäten interagieren, ohne auf einem intentionalen Bildakt zu beruhen. ${ }^{1}$ Von optischen Systemen selbständig erzeugt, registrieren sie Bestehendes, interpretieren Reales. Sie interferieren mit der vertrauten visuellen Welt, formen eine eigene Version von Wirklichkeit, angesichts derer die gängigen Kommunikations- und Interpretationsmodelle an ihre Grenzen stoßen. Es entstehen fremd erscheinende Evidenzen, die gleichzeitig eine eigentümliche Faszination ausüben. Das untenstehende Bild (Abb. 1) resultiert aus einem solchen automatischen Bildgebungs-Prozess, wie ihn Google Street View, die umfassende fotografischen Kartographierung von Städten, initiiert. ${ }^{2}$ Das Bild zeigt eine Frau, den Kopf der Kamera zugewandt. Ob sie bemerkt, dass sie fo-

1 Der auf den ersten Blick abwegigen Idee der Bilder als »Lebensformen « hat sich W. J. T. Mitchell in einem eigenen Buch: »Das Leben der Bilder « gewidmet, in dem er davon ausgeht, dass Bilder "so etwas wie eine Lebensform darstellen « (Mitchell 2008: 22), bei dem das »Leben « von Bildern dem »Modell des Lebens von Tieren und anderen Lebewesen [...] nachgebildet werden kann « (Mitchell 2008: 228). Jacques Lacan imaginierte eine Zukunft, in der fotografierende Maschinen das verlassene Paris bevölkern (Lacan 1991: 64). Die letzten Menschen hatten sie eingerichtet. Diese Maschinen hätten die Sehweise der Menschen angenommen und würden sie in einem fort weitergeben: Das menschenleere Paris ist von photomechanischen Lebensformen bevölkert. Aber auch Horst Bredekamp fragte jüngst angesichts der Mächtigkeit und Nicht-Reduzierbarkeit von Bildern, »warum ein künstliches Gebilde zu leben und zu handeln vermag" (Bredekamp 2010: 165).

2 Die politischen und ethischen Fragen um Googles Street View, der visuellen Kartographierung von Städten, haben einigen Wirbel verursacht, vgl. jüngst etwa die Arbeit von Stephan Doesinger (2012). Inwiefern der Stadtraum damit kontrollierbar gemacht und einer ökonomischen Logik unterworfen wird, wirft bedeutsame Fragen auf. Hier gibt es indessen um die Frage der Interpretierbarkeit automatischer Prozesse, was exemplarisch anhand dieser Technologie gezeigt werden soll, welche in die alltägliche Welt eingreift. 


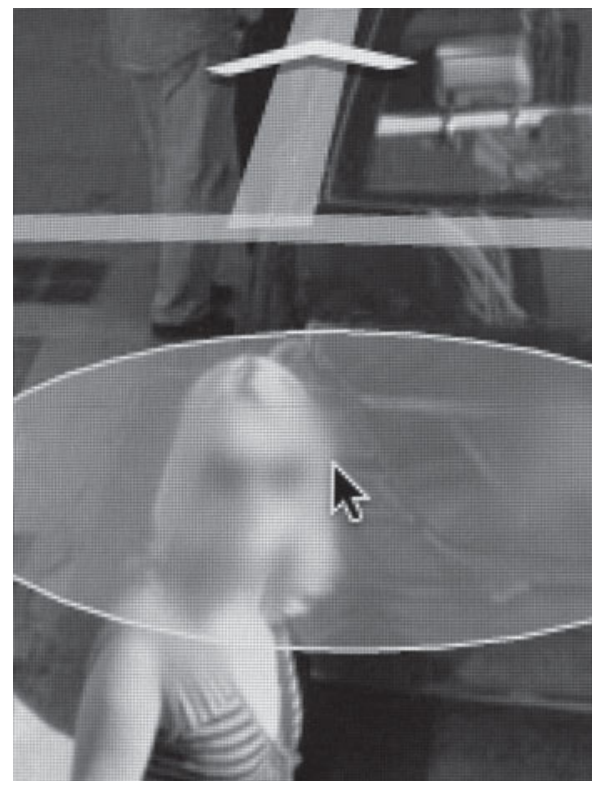

Abbildung 1 Ausschnitt aus einer Pariser Straßenszene, generiert durch Google Street View und abgelichtet von Michael Wolf für sein Projekt »paris street view «.

tografiert wird, lässt sich nicht sagen. Sie trägt eine Brille, ihr Gesicht erscheint merkwürdig verwischt hinter einer weißen, ovalen Fläche. Bei genauerem Betrachten lässt sich der Ausschnitt des Straßenpflasters erkennen, ein Teilstück des Hecks eines Automobils, eine Figur im Hintergrund, die mit Beinen und Unterleib, männlich gekleidet, auch noch in die Szene geraten ist. Hinter der Frau steht ein Personenwagen, seine Frontscheibe und das Heck spiegeln Aspekte der Szenerie, unter anderem ein weiteres Automobil. Die Elemente scheinen zufällig angeordnet: die typische Form eines Schnappschusses.

Diese Bildebene verlangt noch keinerlei Aufmerksamkeit, erscheint als unmittelbar verständlich. Der Titel der Fotografie wie auch die Insignien, Fahrzeug, Straßenabschnitt, Menschen, die scheinbar zufällig ins Bild geraten, rufen eine umfassende Tradition der Fotografie in Erinnerung, die Street Photography: ${ }^{3}$ Die schnappschussartigen Fotografien alltäglicher Straßenszenen, vorübergehender Menschen, zeigen quasi-natürliche Szenerien moderner, urbaner Gesellschaften. Aufnahmen dieser Art durchziehen Zeitungen, Magazine, Filmszenen, private Archive, avancierten gar zu einer eigenen Kunstform, wie etwa in den Bildern Beat Sträulis oder John Millers.

3 Vgl. exemplarisch Scott (2007). 
Doch: lässt sich dieses Bild der jungen Frau überhaupt noch als Fotografie bezeichnen? Es befinden sich weiße Elemente auf diesem Bild, die bei einer klassischen Fotografie nicht auftauchen; eine weit entfernte Ähnlichkeit stellen allenfalls Artefakte von Lichteinstrahlungen auf die Linsen dar, an die sich Betrachter ebenso gewöhnt haben wie an die Ästhetik des Schnappschusses. Das Gesicht der Frau ist von einer ovalen Scheibe überdeckt, wie aus milchigem Glas. Über der Frau lassen sich zwei senkrecht zueinander stehende weiße Balken erkennen, die sich an einer Stelle überlappen, was sich an der dichter erscheinenden Tönung erkennen lässt. Eine gewinkelte weiße Fläche, farblich deutlicher abgesetzt, formt einen der Balken zu einem Pfeil. Eine weitere pfeilartige Struktur, die einem Cursor auf einem Computerbildschirm entspricht, befindet sich direkt in der Mitte des Ovals, scheint das Gesicht beinahe zu berühren, weist aber an ihm vorbei. Das Gesicht selbst wirkt merkwürdig verwaschen, viel stärker, als dies das Oval erwarten ließe. Die Herstellung dieses Bildes lässt sich nicht mehr auf eine fotomechanische Beziehung zu einer stattgefunden habenden Realität reduzieren. Wem oder was ähnelt dann dieses Bild? Was repräsentiert dieses Bild? Lässt sich die Frage einer äußeren Wirklichkeit im Bild angesichts der Synthese verschiedener fotografischer und computertechnischer Elemente überhaupt noch stellen ${ }^{4}$

Immerhin wurde auf die letzte Frage schon eine Antwort formuliert: Ähnlichkeit mit etwas wirklich Existierendem, so Nelson Goodman in Anschluss an Gombrich, ist nicht notwendig ein Kriterium der pikturalen Repräsentation, dass das Bild also das Bild von etwas ist (etwa Picassos Darstellung einer Frau mit Gitarre), darin sich etwas zeigt, und das Bild im weiteren Sinn als Wirklichkeitsdarstellung funktionieren kann (vgl. Goodman/Elgin 1989: 152f.). Die Antwort auf die Frage, was diesem konkreten Bild in Abb. 1 ähnelt, erweist sich freilich als schwieriger: Die Fotografie präsentiert dem Betrachtenden zunächst Insignien einer Straßenszene, und es zeigt zugleich Elemente, die der alltäglichen visuellen Wahrnehmung städtischer Szenen fremd sind. Diese Elemente werden übereinander gelagert und bilden so ein neu gefügtes optisches Ganzes. Dahingehend ist es zumindest das Bild einer Straße, eines Ortes, eines virtuellen computertechnisch erzeugten Kartenbildraumes und der Tätigkeit eines forschenden Benutzers vor dem Bildschirm. Kommuniziert es etwas? Wer oder was kommuniziert dann? Wer ist der »Sender « dieses Bildes und wer der »Empfänger«? Welche Perspek-

4 An sich ließe sich auch von "Montage « sprechen: einer Kulturtechnik, »angefertigtes Material so zusammenzusetzen, dass ein neues Produkt entsteht «(Stiegler 2009: 289). Lev Manovich insistiert aber darauf, angesichts von Computerbildern den Begriff der »Synthese « zu verwenden. Bei üblichen Computerbildern handelt es sich um eine Kombination visueller Elemente, die auf die Synthese eines kontinuierlichen Wahrnehmungsraum zielen, im Gegensatz zur künstlerischen Form der Montage, die eher die Fragmentierung der Welt zum Ausdruck bringt (Manovich 2001: $141 \mathrm{ff}$.). 


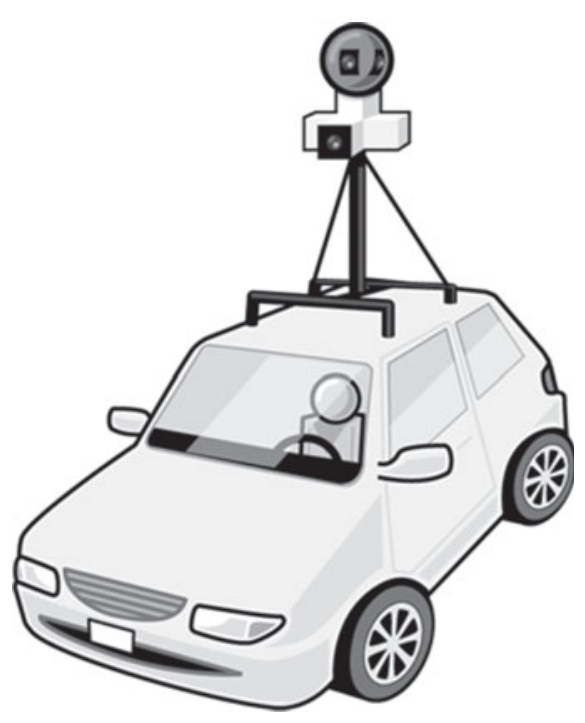

Abbildung 2 Ein Interpret der urbanen Welt. Googles Street View-Gefährt

tive vergegenständlicht das Bild: jene der fünfzehn Kamera-Objektive auf dem Dach von Googles Automobil, das durch die Straßen fuhr (vgl. Abb. 2), oder jene des Anwenders, der den damit erzeugten digitalen Bildraum für seine Zwecke gebraucht?

Lässt sich die visuelle Evidenz vielleicht unter dem Aspekt eines »Mediums « begreifen, als etwas, das qua Begriff >in der Mitte < liegt, das überträgt (Krämer 2008: 9 f.)? Zwischen Betrachter und Straßenszene? Zwischen Computersystem und Betrachter? Oder ist der tätige Benutzer nicht eher das Medium, der Vermittler zwischen computertechnischem Bildraum und städtischem Raum, der durch seine Gebrauchsspuren neue Daten erzeugt? Das vorliegende Bild scheint sich den etablierten Kategorien der Erfassung und des Verständnisses von Bildlichkeit merkwürdig zu entziehen. Und dennoch beinhaltet es Ähnlichkeitsbeziehungen zur Außenwelt, insofern, als dass es einem visuellen System entstammt, das dazu da ist, einen Benutzer durch eine Stadt zu führen, insofern auch, dass es eine konkrete Person zeigt, die im Prinzip so wirklichkeitsgetreu registriert wurde, dass ihr Gesicht unkenntlich gemacht werden musste, damit sich die reale Person >dahinter $\ll$ nicht identifizieren lässt.

Aufgenommen ist das Bild von einer Roboterkamera, die sich auf einer der Fahrzeuge von Googles Street View befindet - ergänzt durch Laser zur Distanzerfassung, GPS-Gerät und Bewegungssensoren. Zwar haben Ingenieure das Fahrzeug und die Kamera konstruiert, das Suchmaschinenunternehmen entwarf die 
technischen Pläne. Aber es ist keine menschliche Intention an der vorliegenden Aufnahme beteiligt, die konkrete Szene und Person genau auf diese Weise aufzunehmen, es ist keine Suche nach dem `rechten Augenblick « auszumachen, der für den Fotografen, wie Cartier-Bresson schrieb, genau und nur dann gelingt, »wenn der Fotograf selbst Bestandteil des gesellschaftlichen Lebens ist « (Cartier-Bresson 1998: 38). Bestandteil des städtischen gesellschaftlichen Lebens im Falle des vorliegenden Bildes war der optische Automat, durch die Stadt gefahren von einem Chauffeur, der sich wohl auf eher auf den Verkehr konzentrierte. Automatisch erfolgte die Verwischung des Gesichts, das aufgrund von Algorithmen erkannt wurde, automatisch wurden weitere Bestandteile des Bildes hinzugerechnet, als Reaktion auf den Gebrauch des Bildraumes auf dem Computer selbst, um dem Anwender zu helfen, sich im virtuellen Bildraum zu bewegen und sich je nach Gelegenheit im realen Raum zu orientieren.

Freilich, bei diesem Bild handelt es sich nicht um irgendeine Fotografie; es wurde vom Fotografen Michael Wolf aus dem Bildraum von Googles Street View ausgewählt. ${ }^{5}$ Wolf lichtete den Ausschnitt des fotografierten Raumes, wie er ihm auf dem Computer-Bildschirm erschien, wiederum mit seinem Foto-Apparat ab. Digitale Punkte des Bildschirms werden zu digitalen Punkte der Kamera übersetzt. Der Bildschirm, auf dem ein Ausschnitt eines computertechnischen wie fotografischen Bildraumes erscheint, bildet das fotografische Objekt. Street View, so scheint diese Anordnung zu besagen, bildet ganz augenscheinlich weniger einen Ersatz des Sehens als ein Neu-Hinzukommen technischer Anordnungen, welche die Wahrnehmung von Städten auf besondere Weise erweitert. Vor einiger Zeit erkannte Jonathan Crary in seiner Untersuchung zur Geschichte des Sehens und Beobachtens in solchen automatischen, computergestützten und -generierten Arten der Bilderzeugung breitere technologisch-soziale Transformationen, die »das Sehen auf einer vom Betrachter und vom menschlichen Auge getrennten Ebene neu anordnen. « Es zeichne sich ab, dass » die neu entwickelten Techniken der Bildproduktion zu dominanten Visualisierungsmodellen werden « und mehr noch »die wichtigsten sozialen Prozesse und Institutionen bestimmen." Dahingehend artikuliert sich für Crary angesichts dieser automatisch erzeugten Bilder auch ein Gefühl des Unverständnisses, der Fremdheit, denn die »unerbittlich fortschreitende Abstraktion des Visuellen « von den gängigen Formen des Sehens wird durch technische Erklärungen nur unverständlicher und in eine Aura des Geheimnisvollen gehüllt « (Crary 1996: 12; Hervorhebung F. K.).

Diese unfassbare Aura der automatisch erzeugten Bilder wird auch von Vilém Flusser erkannt. Er spricht von einer » magischen Faszination technischer Bilder «,

5 Dokumentiert ist das Projekt des Fotografen Michael Wolf, auf das später noch eingegangen wird, auf http://www.photomichaelwolf.com/paris_street_view/. 
Abbildung 3 Modernes Bilderrätsel: Hund mit zwei Schatten. Michael Wolf street view: a series of unfortunate events \# 17.

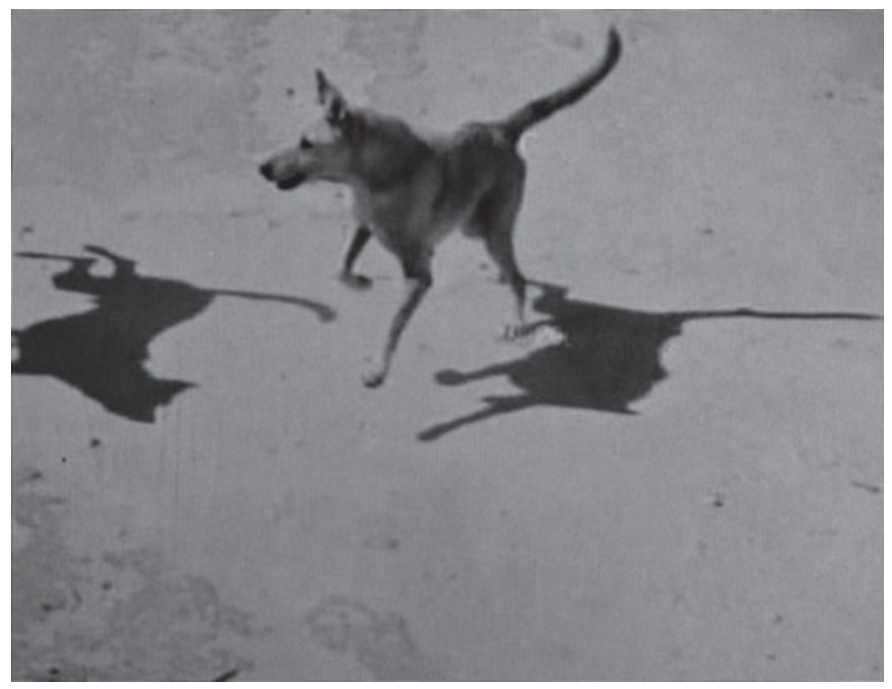

die gerade daher rühre, dass ihre Erstellungsbedingungen, im Gegensatz zu anderen Bildern und Symboldarstellungen, in einer "Black Box " gründen (Flusser 1997: 15 f.). Desgleichen schreibt Paul Virilio über automatisch erzeugte Bilder, dass sie - von Maschinen erstellt, von Maschinen bearbeitet und interpretiert dem direkten Blick nicht mehr zugänglich sind, für »uns zu einem Äquivalent dessen « werden, "was jetzt schon die gedanklichen Bilder eines fremden Gesprächspartners sind ... ein Rätsel « (Virilio 1989: 137; Hervorhebung F. K.). Doch weshalb werden die Bilder in eine "Aura das Geheimnisvollen gehüllt «, wenn Ingenieure rational nachvollziehbare technische Pläne der Maschinen entworfen und Informatiker die Zeilen ihres Codes programmiert haben? Wenn sie also nichts weniger als Ausdruck höchster technischer Rationalität sind? Die These lautet: gerade weil die Wahrnehmungsweisen der sichtbaren Welt von optischen Maschinen wie Menschen nicht bezugslos zueinander stehen, sondern beide auf Interpretationen der sichtbaren Welt beruhen. Interpretationen, die aber nur begrenzt zueinander kompatibel sind, erzeugen angesichts der automatischen Bilder Rätsel.

Mit anderen Worten gesagt, das Mysterium solcher Bilder (ein Beispiel mag Abb. 3 liefern) lässt sich gemäß der hier vertretenen Auffassung zumindest zu einem Teil über die Annahme erhellen, dass jenseits der Grenzen des menschlichen Interpretationsvermögens, dort wo Fotomechanik und Computertechnik 
an der Registrierung der Wirklichkeit arbeiten, nicht totes Maschinengelände liegt, ein rohes Feld des Nichtinterpretierten, sondern sich andere Formen der Interpretation finden, die gerade deshalb ein Mysterium bilden, weil sie nicht vollständig verstehbar sind, im Gegensatz zu einem einfachen mechanischen Ablauf. Um diese These zu verfolgen, erweist es sich aber zunächst als notwendig, die Differenz der Street View-Fotografien zur klassischen Form der Fotografie herauszuarbeiten, insbesondere hinsichtlich ihrer Bezüge zu einer äußeren Wirklichkeit. Vor diesem Hintergrund lässt sich in einem zweiten Schritt die automatische Interpretationsleistung verstehen und in einen Bezug zur menschlichen Form der Interpretation stellen.

\section{Die Frage nach dem Fotografischen in Googles Streetview-Bildern}

Google Street View fotografiert Städte, darin steht das Projekt in einer langen fotografiehistorischen Tradition. Doch die erhaltenen Fotografien werden zusammengerechnet und automatisch nachbearbeitet. Daraus entstehen Bilder der städtischen Szenerie, die noch nie gesehen waren: eine neue fluide Bildform, die aber dennoch mit der Realität der Städte auf irgend eine Weise in Verbindung steht, bei der der Wirklichkeitsbezug so stark wirkt, dass er in einem zweiten Schritt vernichtet werden muss. Doch was auf diesen Bildern zu sehen ist, interpretiert Google selbst unterschiedlich. Im Jahre 2009 schrieb Google: »Street View enthält Bilder, die genau dem entsprechen, was Sie auf der Straße sehen [...] Unsere Bilder zeigen das an, was mit unseren Fahrzeugen an bestimmten Orten zu einem bestimmten Zeitpunkt aufgenommen wurde..$^{6}{ }^{*}$

Wirklich lässt sich die Fotografie eines Taxifahrers aus einer Serie von fotografischen Impressionen der Stadt Zürich (Perrottet 2011) mit dem von Googles Street View gelieferten Bildraum überraschend deutlich rekonstruieren (vgl. Abb. 4). Bei der Aufnahme des Taxifahrers tragen die Menschen Mäntel, es hat geregnet, das Graffiti an der Wand erscheint frisch. In der Street View-Fotografie zeigt sich das Graffiti jedoch ausgebleicht, die Fensterläden stehen nun offen, die Straße ist trocken, die Kleidung, die das Mädchen mit verwischtem Gesicht trägt, signalisiert einen heißen Sommertag. Was heißt nun "genau dem entsprechen, was Sie auf der Straße sehen «? Die Architektur, die Perspektive auf die Stadt lässt sich rekonstruieren, aber nicht die konkrete Szenerie, die Stimmung. Dieser Umstand zwingend

6 http://www.google.ch/help/maps/streetview/privacy.html, abgerufen am 13. Dezember 20o9, noch rekonstruierbar über: web.archive.org. 
Abbildung 4 Straßenszene aus Zürich: Einmal aus dem Fotoband »Taxi« des fotografierenden Taxifahrers Oliver Perrottet (Perrottet 2011), ein andermal rekonstruiert aus Google Street View (rechts).

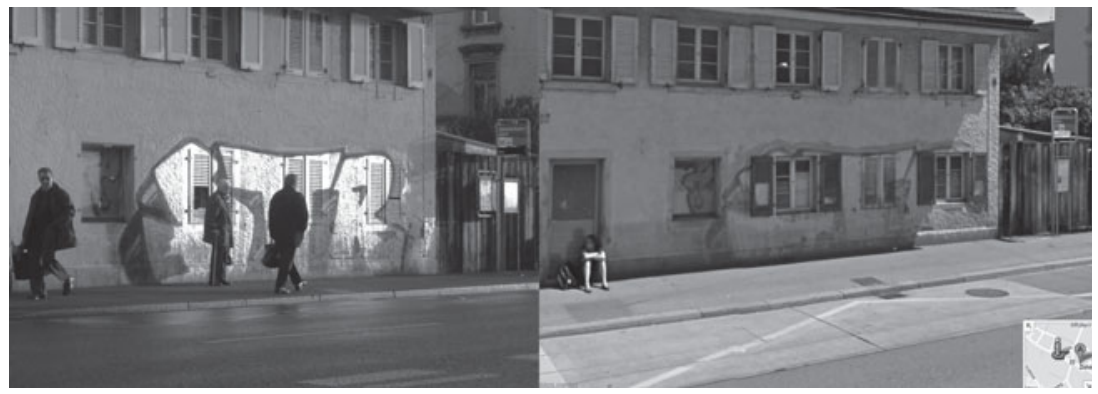

auftretender Differenzen bei erstaunlicher perspektivischer Identität lässt Google seine Erklärung dessen, was gesehen wird, alsbald ändern.

"Street View enthält Fotos, die dem entsprechen, was jedermann auf der Straße sehen kann (Hervorhebung F. K.) «, heißt es zweieinhalb Jahre später. Die sprachliche Verschiebung ist nicht bedeutungslos. In der ersten Version (Fotografien, »die genau dem entsprechen, was Sie auf der Straße sehen «) erscheint die Kamera gleichsam als Ersatz des konkreten individuellen Sehakts auf der Straße. Die Perspektive der Maschine wurde der Perspektive eines realen Menschen gleichgesetzt. Die zweite Version erhebt diese Gleichsetzung des maschinellen und individuellen Sehaktes wieder auf: Zum einen wird der Bezug dessen, was auf den Bildern ist, zur Möglichkeitsform erhoben; zum anderen wird das potentiell »Erblickbare « von dem konkreten individuellen Sehakt abstrahiert: die personale Form gerät zum Jedermann, zum abstrakten Sehsubjekt, zum Blick des "generalisierten Anderen «. Street View wird nun definiert als abstrakter, apersonaler Raum der Sichtbarkeit, der eine transparent sichtbare aber virtuelle Stadt enthält, welche die reale verdoppelt und sie auf neue Weise dem Schauen verfügbar macht.

Dennoch spricht Google noch von » Fotografien «, also von singulären, historischen Ereignissen in der konkreten Raum-Zeit. Die Bilder, wie sie letztlich durch den Gebrauch von Street View generiert werden, brechen aber an entscheidenden Stellen mit dem, was sich als ein Paradigma des Wirklichkeitsbezugs von Fotografien beschreiben lässt. Roland Barthes hatte in einer bekannten Stelle der Hellen Kammer noch davon gesprochen, es lasse sich "nicht leugnen, dass die Sache dagewesen ist «, die auf der Fotografie erscheint: fotografische Referenten seien »notwendig reale Sache[n], die vor dem Objekt platziert war[en] und ohne die es keine Photographie gäbe (Barthes 1989: 86; Hervorhebungen im Original). 
Die Fotografie, wie Rosalinde Krauss schreibt, setzt im gängigen Sinn eine Einheit der Realität voraus, auf die sie Bezug nimmt, als »dessen-was-zur-gleichenZeit-präsent-war " (Krauss 1998: 114). André Bazin wiederum schrieb in seinem berühmten Aufsatz zur Ontologie der Fotografie: »Welche kritischen Einwände wir auch haben mögen, wir sind gezwungen, an die Existenz des wiedergegebenen Gegenstands zu glauben, der ja tatsächlich wiedergegeben, das heißt in Raum und Zeit wieder gegenwärtig gemacht wird « (Bazin 2004: 37). Die "Metaphysik des Referenten, dass die Sache da gewesen ist « (Dubois 1998: 52), erweist sich aber im eingangs gezeigten Bild als merkwürdig obsolet, ohne ganz zu verschwinden. Niemand wird vor der Busstation »Zehntenhaus « in Zürich einem Mädchen mit verwischtem Gesicht begegnen, niemanden einer jungen Frau in Paris, über der sich geometrische Figuren erheben. ${ }^{7}$ Der repräsentationale Aspekt ist in den Street View-Bildern im Hinblick darauf, was zur gleichen Zeit im Fokus der Kamera erschien, zerstört, denn die computertechnischen Einrechnungen waren ja vor ihrem Gebrauch nicht existent, und auch das verwischte Gesicht ist rein künstlicher Natur. Der konkrete Wirklichkeitsbezug bleibt indes erhalten, der Ort, den der Bildausschnitt zeigt, muss sich in der urbanen Realität tatsächlich finden lassen.

Genauso wenig wie eine Ontologie oder ein fotografischer Realismus vermag ein anderes epistemologisches Paradigma die Wirklichkeit der Street View-Bildwelt zu fassen: die rein kulturorientierte Auffassung, aufgrund derer eine Fotografie als Effekt einer »arbiträren Gestaltung « erscheint, die sich gleichsam aus einem vorhergehenden kulturellen oder ikonographischen Sinn ergibt, einem Sinn, für den die Fotografie nur ein Dokument darstellt, indem er sich abschattiert (Dubois 1998: 46). Gerade bei der schieren Menge von Evidenzen einer fotografierten Straßenszene lässt sich fragen, ob sie sich in ihrer Fülle als Effekte einer kulturellen Codierung des Bildes begreifen lassen. Als ähnlich unbefriedigend erweist sich auch die Übertragung der Auffassung Crarys über das rein technische Bild auf die Fotografien von Street View, nämlich dass, »wenn man überhaupt sagen kann, dass diese Bilder etwas bezeichnen oder sich auf etwas beziehen, dann wohl auf Millionen bits elektronischer mathematischer Daten « (Crary 1996: 12). ${ }^{8}$ Die gezeigten Google-Fotografien bezeichnen ganz klar die Realität einer Straßenszene in Paris oder Zürich, und der ökonomische Wert (für Google) und der Ge-

7 Stephan Günzel (2011: 73) diskutiert allerdings Fälle, in denen Fans von Computerspielen sich selbst als ihre eigenen Avatare verkleiden, mit allen dazugehörigen virtuellen Gegenständen, um sich damit im reellen Raum zu zeigen. So besteht also die Chance, dass man sich plötzlich martialische Krieger vor dem Kölner Dom mit überdimensionierten > Waffen aus Schaumstoff gegenüber sieht. Das verwischte Gesicht erscheint allerdings als beinahe unmöglich in die Realität zu übertragen.

8 Desgleichen auch Doesinger (2012: 48): Das digitale Bild repräsentiere Bezugspunkte, die wiederum »nur Repräsentationen von Bezugspunkten « seien. 
Abbildung 5 Gombrich schrieb über das Bild links: „Hier nahm der Photograph zwei verschwommene Gestalten vorübergehender Kinder mit auf. So etwas hätte noch vor nicht allzu langer Zeit Verleger und Leser schockiert « (Gombrich 1984: 271). Ganz andere, noch nicht einfach zu begreifende Gestalten erscheinen in den automatisch erzeugten Bildern von Google Street View (Michael Wolf, paris street view \#7).
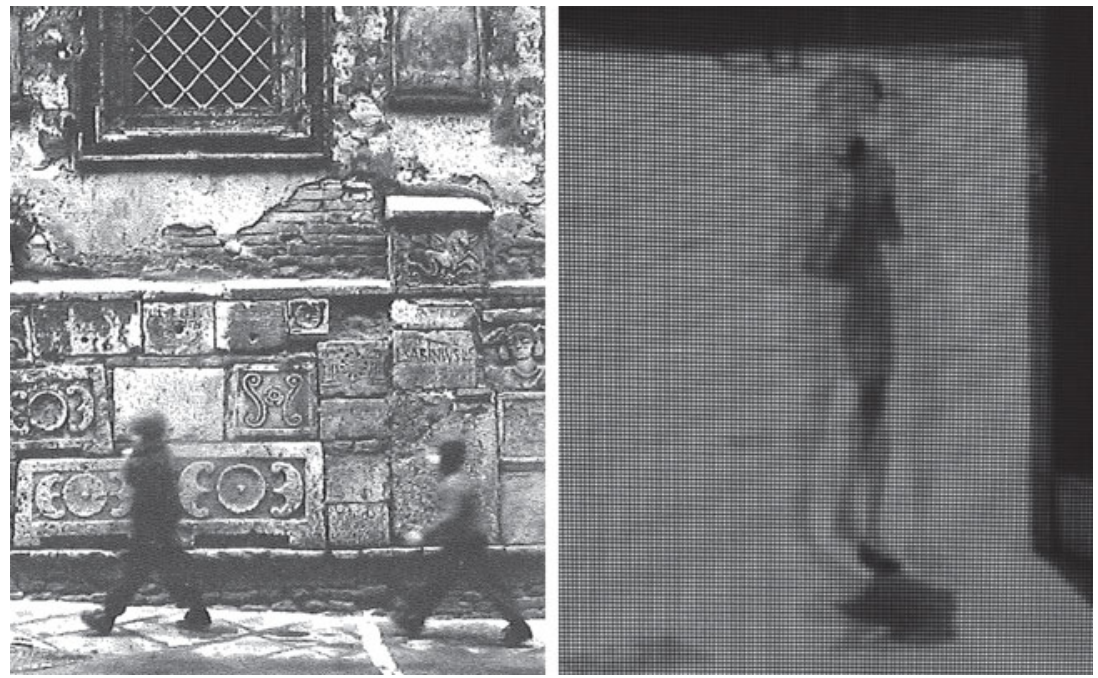

brauchswert für den Benutzer des Bildes ergibt sich gerade daraus, dass sich ein Betrachter in der Realität der Straßenszenen orientieren kann, ansonsten das Projekt schlicht vergessen ginge.

Wie lässt sich nun aber begreifen, auf welche Weise das Bild urbane Wirklichkeit trägt und erzeugt, ohne von einer Metaphysik der Referenten, einem übergeordneten Sinn oder aber einer rein technischen Selbstbezüglichkeit auszugehen? Lässt sich von Wirklichkeitstreue trotz aller Konstruiertheit sprechen? Eine Wahrnehmungstheorie wie sie Gombrich formulierte, sieht bereits das Erkennen von Realität, wie sie in den klassischen Fotografien von Straßenszenen zum Ausdruck kommt, keineswegs als gegeben an: "Noch vor ein, zwei Generationen ", schreibt Gombrich, »erklärte man aufs Geradewohl geknipste Schnappschüsse für unverständlich «, Schnappschüsse, wie Abbildung 5 einen zeigt. Das »Publikum hatte damals «, so Gombrich, schlicht »noch keine Vorstellung von dem Zusammenhang zwischen den Bildern und dem Prozess, durch den sie zustande kamen. (Gombrich 1984: 269). Um Aspekte des Wirklichen in Fotografien zu erkennen, bedürfe es eines Wissens, das sich nicht durch flüchtiges Hinschauen 
erwerben lässt: Kenntnis der "pikturalen Konventionen « und der "referentiellen Beziehungen « ist hierzu erforderlich, aufgrund deren es erstellt wurde (Goodman/Elgin 1989: 154), aber auch die Vertrautheit mit den bildlichen Konventionen und Einsicht in die konkrete Technik der Bildproduktion. Oder anders ausgedrückt: Weniger die »Eigenschaften des Bildes selbst als das Verständnis der Technik « bilden die Voraussetzung dafür, dass » Bilder `gesehen ` werden können « (Gombrich 1984: 269).

Diese kognitiven Anpassungsleistungen an die Techniken des Bilderzeugens als Voraussetzungen, überhaupt etwas in den Bildern zu >sehen weitgehend dem Bewusstsein entzogen. »Wenn wir ein pikturales System beherrschen «, also all jene Techniken und Praktiken, die das Bild erzeugt haben und nicht nur das Bild selbst, sehen wir zunächst aber einfach, »was seine Bilder repräsentieren «; der technische Hintergrund ist ins Unbewusste gerutscht, so Goodman und Elgin in Anschluss an Gombrich. Der Wahrnehmungsprozess verlaufe "so automatisch, dass wir gern vergessen, dass Interpretationen stattfinden " (Goodman/Elgin 1989: 154; Hervorhebung F. K.). Das heißt, notwendig zur Identifikation von Wirklichkeitsbezügen in Bildern sind nicht nur Praktiken des Einübens, mittels derer visuelle Evidenzen eines Bildes mit Aspekten der Realität verknüpft werden können, sondern notwendig ist auch implizites Wissen über die technischen Arrangements der Bilderstellung. Oder anders ausgedrückt: Wenn Reales in der Fotografie erkannt wird, wenn eine Wirklichkeit in den Fotografien gesehen ist, so geschieht dies nicht bloß aufgrund einer automatischen Interpretation des fotografischen Abzugs, der Fotografie als Ding, sondern gleichzeitig aufgrund der impliziten Interpretation des ganzen technischen Umfeldes, des "pikturalen Systems « selbst, also der Maschine der Repräsentationserzeugung. Es wird, so ließe sich folgern, nie bloß ein Bild gesehen und interpretiert, sondern immer gleichzeitig auch die technische Konfiguration, die es erzeugt hat. Gerade deshalb entstehen immer auch Grenzen des Sehmöglichen, Fragen nach der Wirklichkeitstreue. Eine Abbildung, die gleichzeitig das Gesicht von der Vorder- wie der Rückseite zeigt, bildet ohne Bearbeitung eine schiere Unmöglichkeit im optischen System der Fotografie. Trotz aller technischen Konstruiertheit, gibt es also Kriterien der Wirklichkeits treue oder anders ausgedrückt: Entsprechungen zu einem urbanen Korrelationsraum, ohne dass jeder Bildbestandteil ein Pendant im realen Raum besitzen müsste. 


\section{Eine neue Vision des urbanen Raums}

Die digitalen Bilder beinhalten zweifelsohne Aspekte der äußeren Wirklichkeit im Sinne Gombrichs, oder des Indexalischen im Sinne Dubois' (vgl. Dubois 1998: 62). Sie verkörpern ein Dispositiv, das Spuren äußerer Effekte transformiert, auch wenn die Bilder digital erzeugt und bearbeitet wurden. Mehr noch, der Wirklichkeitsbezug ist bei aller Automatik und computertechnischen Künstlichkeit so umfassend, die erfasste Wirklichkeit drängt so nachhaltig in den Bildraum, droht sich dort mit anderen Wissensformen anzureichern (über die Personen und Lokalitäten), dass dieser Wirklichkeitsbezug über Verwischungstechniken wieder ausgelöscht werden muss: Gesichter und Autokennzeichnen verwischt und Hausfassaden unkenntlich gemacht werden. Gleichzeitig, wie gesehen, sind die Bilder auch hoch artifiziell, indem computertechnische Bildelemente hineingerechnet werden und über die Verrechnung der Bilder miteinander Perspektiven auf den urbanen Raum entstehen, die zuvor unmöglich waren (und u. U. zu eigentümlich verzerrten Raumbildern führen). Die Erzeugung einer neuen Version der urbanen Welt, die damit gelingt, geschieht einerseits über fotografische Realitätsnähe und gleichzeitig über die Negation von zentralen Aspekten dieser Realität selbst, was wiederum eine Interpretation dieser Realität notwendig voraussetzt. Wie kann diese Arbeit an der Realität auf automatische Weise funktionieren?

Google setzt Algorithmen ein, um Bezüge zur realen Welt, zum realen geschichtlichen Augenblick der Straßenszenen zu löschen. Aber es stellt sich selbst für den Konzern die Frage, was denn eigentlich genau geschieht, wenn er die Algorithmen zur Verwischung von Erkenntlichkeit autonom rechnen lässt. In der zuvor vorgestellten Beschreibung von Googles Street View hieß es zunächst: »Wir haben ein hochmodernes Tool entwickelt, um Gesichter und Autokennzeichen auf allen Street View-Bildern unkenntlich zu machen. Wenn auf einem unserer Bilder ein erkennbares Gesicht, wie z. B. ein Passant auf dem Gehweg oder Autokennzeichen abgebildet sind, werden diese mithilfe des Tools automatisch unkenntlich gemacht, um eine Identifizierung auszuschließen. Falls versehentlich etwas übersehen wurde, können Sie uns dies melden. «9

Auch dieser Abschnitt wurde in einer weiteren Version verändert, um der nachhaltigen Kritik an der Verletzung der Privatsphäre im öffentlichen Raum entgegenzutreten. Später, in der nachfolgenden Version, ist zu lesen: »Wir verwenden weder Film- noch Videomaterial, sondern ausschließlich Fotos. Street View ist vielmehr die Darstellung von Straßenbildern, die der Orientierung dienen sollen. Da die Bilder bei Tageslicht angefertigt werden müssen, um eine ausreichende

9 http://web.archive.org/web/20091213031253/http://www.google.ch/help/maps/streetview/ privacy.html. 
Qualität zu erreichen, lassen sich zufällige Aufnahmen von Passanten nur sehr schwer vermeiden. Bei der Entwicklung von Street View nehmen wir Ihre Privatsphäre sehr ernst. Deshalb werden Gesichter und Autokennzeichen auf den Street View-Bildern, wo sie erkennbar sind, automatisch von unserer Technologie noch vor der Veröffentlichung unkenntlich gemacht. «10

Die Modifikation der Bilder erhält in der neuen Formulierung eine Begründung und eine Rechtfertigung: die Realität der Straßenszenen, die sich in die Fotografie einbringt, ist teils ein ungewünschter Nebeneffekt, aufgrund des Bemühens, so wiederum die Rechtfertigung, »Straßenbilder « zu schaffen, die der Orientierung dienen sollen. Diese unerwünschten Realitätseffekte belebter Straßen, die der Identifikation sozialer Realitäten nicht aber der Orientierung dienen, müssen entfernt werden, um die so reduzierten Straßenbilder wiederum mit anderen Informationen (beispielsweise über kommerzielle, verkehrstechnische und touristische Begebenheiten) anzureichern. Doch um diese potentiell korrumpierenden Informationen erkennen zu können und dadurch eine neue, bereinigte Form des urbanen Raums zu erzeugen, ist das Unternehmen auf eine Interpretation seiner Bilder angewiesen, denn bei den Aufnahmen, wie bei der Fotografie an sich, handelt es sich, wie sich Dubois markant ausdrückt, um ein "Dispositiv der automatischen Einschreibung ", das sich selbst nicht interpretiert, nicht kommentiert, sondern "stumm und nackt « ist, "platt und dumpf « (Dubois 1998: 87). Die systematische Veränderung des Realen verlangt auf jeden Fall eine vorhergehende Auslegung: des Trennens von Erwünschtem von Nicht-Erwünschtem. Es ist aber aufgrund der schieren Fülle des Materials unmöglich, dass ein Mensch all diese Interpretationsarbeit vornimmt, entsprechend müssen es Maschinen sein, die Evidenzen feststellen, und sie daraufhin verändern. Worin unterscheiden sich die maschinellen Interpretationsformen von den menschlichen und worin sind sie identisch? Ist es überhaupt zulässig, von Interpretationen zu sprechen? Gibt es jenseits der Grenzen der menschlichen Interpretation nicht auch andere Interpretationsformen? Und wo überschneiden sich die beiden Formen?

\section{Was heißt »automatische Interpretation «?}

Wenn per Definition nur menschlichen, vernunftbegabten Wesen Interpretationsleistungen zugesprochen werden, die das kunstvolle hermeneutische Verstehen der Sache meint, dann interpretieren Maschinen die Welt selbstverständlich nicht. Die hier vorgetragene These besagt, dass es sich lohnt, den Interpretationsbegriff nicht den Menschen vorzubehalten sondern davon auszugehen, dass es verschie- 
dene Formen der Interpretationen gibt, die beständig zwischen menschlichen und maschinellen Instanzen übersetzt werden. Die etymologischen Spuren im Interpretationsbegriff legen bereits nahe, ihn auf maschinelle Bereiche zu erweitern. >Interpretation ‘ leitet sich nicht nur von deuten, auslegen sondern auch von interpretari, dolmetschen, übersetzen her. Der Interpret war auch Übersetzer und Vermittler. ${ }^{11}$ Die Frage der Interpretation und Interpretierbarkeit öffnet sich entsprechend, wenn Interpretation nicht als bloßer menschlicher Bewusstseinsakt konzipiert ist, sondern erweitert wird auf sprachliche oder semantische Prozesse, die folglich immer auf Übertragung und Übersetzung zielen. ${ }^{12}$ Nicht zuletzt kennt bekanntlich auch die elektronische Datenverarbeitung das Konstrukt des »Interpreters «, Computerprogramme, welche in einer Computersprache geschriebenen Anweisungen (beispielsweise in Pascal) interpretieren und in die Maschinensprache übersetzen. Wenn der Interpretationsbegriff über Anwendung von (semantischen) Regeln begriffen wird, lassen sich menschliche und maschinelle Interpretationen durchaus gegenüber stellen, wie Searle in seinem Gedankenexperiment des Chinesischen Raumes darlegt (Searle 2008). Eigentlich will Searle mit dieser gedanklichen Versuchsanordnung die These der Möglichkeit einer Artificial Intelligence widerlegen, also die Annahme, dass ein Computer das menschliche Verstehen imitieren könne - selbst wenn der Anschein des Verstehens entsteht. ${ }^{13}$ Das Experiment zeigt aber auch, auf welche Weise sich Interpretation und Verstehen getrennt denken lassen. Searle nimmt damit eine entscheidende Differenzierung von Begriffen vor, deren Bedeutung oft nicht unterschieden wird. Sie hilft, die Friktionen der maschinellen und humanen Interpretationsformen zu verstehen. Deshalb sei das Experiment kurz vorgestellt.

Ein Mensch befindet sich in einem geschlossen Raum mit verschiedenen Stapeln von Karten, auf denen chinesische Zeichen vermerkt sind und die gleichzeitig mit Handlungsanweisungen auf Englisch versehen sind, was mit diesen Symbolen zu tun sei. Der Mensch spräche kein Wort Chinesisch. Nun erhält die Person durch einen Briefkasten-Schlitz Karten mit chinesischen Zeichen. Er ordnet sie alleine aufgrund der visuellen Signatur anderen Karten in seinem Raum zu, die gleichzeitig auf Englisch beschriftet sind und verfolgt die englischen Handlungsanweisungen, die darin bestehen, aus einem anderen Stapel mit chinesischen Zeichen, Karten zu ziehen, zusammenzustellen und durch den Schlitz wieder auszugeben. Es handelt sich bei den zweisprachigen Karten also nicht um ein seman-

11 Vgl. die Ausführungen zum Begriff der Interpretation in Mittelstrass' Enzyklopädie der Philosophie und Wissenschaftstheorie (Mittelstrass 1995: 273 ff.). Handkehrum, so selbst Gadamer, ist wiederum jede Übersetzung auch Interpretation (vgl. Gadamer 1986: 342).

12 Vgl. zum Kontext vgl. Stegmüller (1989: 378).

13 In den erkenntnistheoretischen Klüften, die eine solche Frage aufwirft, gräbt auch Umberto Eco in seinem Werk Die Grenzen der Interpretation (Eco 1995, Kap. 4.5). 
tisches Lexikon, sondern um Vorschriften, im Sinne von »wenn dieses Zeichen neben diesem Zeichen, dann tue das «. Nehmen wir an, der Chinese außerhalb des Raumes gäbe eine Geschichte in chinesischen Zeichen ein und stelle Fragen dazu, und die Regeln, wie mit den Zeichen umzugehen sei, seien eindeutig und umfassend kodifiziert, dann wird der Chinese wohl annehmen, dass der Mensch innerhalb des Raumes, Chinesisch verstünde, wenn er alsbald die korrekte Antwortkarte in den Händen hält. In der Tat aber befolgt er nur Regeln, ohne ein Wort Chinesisch zu verstehen. Dennoch interpretiert er die visuellen Zeichen, indem er Handlungsanweisungen vollzieht und auf Zeichen anwendet, er übersetzt Regeln in Handlungsanweisungen, ohne den Sinn der sprachlichen Zeichen erkennen zu können, ohne die zugrundeliegende Sprache in irgend einer Weise zu begreifen. ${ }^{14}$ Ironischerweise scheint gerade diese Kritik der harten Artificial Intelligence-These, nämlich dass eine anscheinend richtig interpretierte Phrase auch Verstehen bedeute, zu erläutern, auf welche Weise Maschinen von Google die Straßenszenen > sinnvoll bearbeiten können. Aufgrund von Algorithmen interpretieren Googles Computer bestimmte Bitmuster als Gesichter und verändern sie, ohne, dass wir und wohl auch die Ingenieure von Google im konkreten Fall genau verstehen, was sie tun. Es entsteht eine neue Version von Darstellungen, als hätten die Automaten die Straßenszene verstanden.

Dieses Anscheinend-Verstehen wird um so faszinierender, als auch Fehlinterpretationen auf verschiedenen Stufen geschehen. Abbildung 6 zeigt zwei Models auf einem Plakat, das heißt, es handelt sich um einen öffentlich präsentierten fotografischen Akt. In den öffentlichen Raum gestellt, wurde das Plakat wiederum von einer automatischen Kamera wahrgenommen, die permanent die Straßen ablichtet, ohne Eingreifen des Menschen selbst. Das Programm sucht nach visuellen Informationen, die es als > Gesicht « interpretiert und modifiziert die digitalen Informationen so, dass die Gesichter als verwischt erscheinen. Die Pixelordnung auf dem Plakat wird als Gesicht eines Passanten betrachtet, weil der Algorithmus den Kontext seiner Darstellung nicht erkennen kann. Diese Bearbeitung von Bitmustern, die als Gesicht interpretiert werden, treibt durchaus seltsame Blüten: allenthalben >versteht ^ der Algorithmus etwas als Gesicht, das keines ist, wie im folgenden Beispiel der Radkappe eines UPS-Lieferwagens in New York, das der Automatismus als faciale Form erkannt hatte (Abb. 6 links). Angesichts dieser offensichtlichen Fehlinterpretationen stellt sich die Frage, ob sich ein maschinelles

14 Searle drückt dies später so aus: » The purely syntactical operations of the computer program are not by themselves sufficient either to constitute, nor to guarantee the presence of, semantic content, of the sort that is associated with human understanding. The purpose of the Chinese Room thought experiment was to dramatically illustrate this point. It is obvious in the thought experiment that the man has all the syntax necessary to answer questions in Chinese, but he still does not understand a word of Chinese " (Searle 2009). 
Abbildung 6 Gesichtsinterpretation in Google Street View. Links offenbar als Gesicht interpretiertes Rad eines Lieferwagens. Rechts: Gesichter von Models auf Plakatwände als Gesichter von Passanten interpretiert
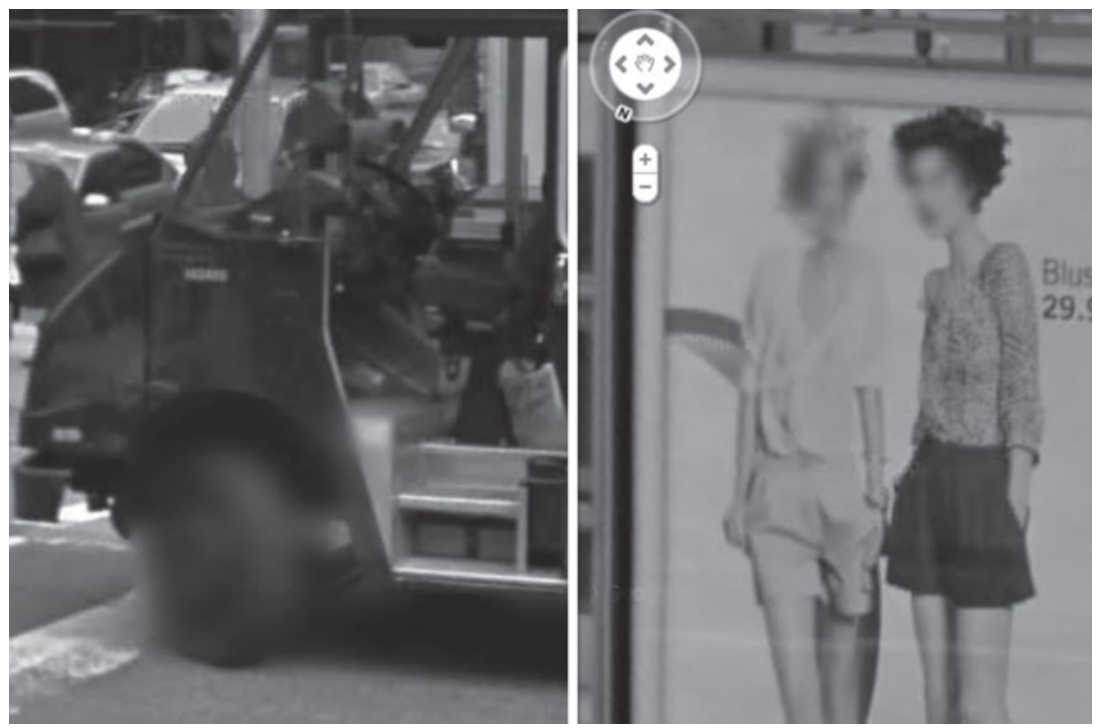

Interpretieren aufgrund seiner Fehleranfälligkeit nicht doch klar vom menschlichen Interpretieren trennen lässt.

Die Frage zu beantworten ist allerdings nicht so einfach. Im Jahre 2005 meinten Gläubige unter einer Schnellstraße eine Marienerscheinung entdeckt zu haben. Hunderte von Katholiken pilgerten zu einer Betonmauer unter der strostlosen « Autobahnuntererführung des "Kennedy Express « in Chicago, um Flecken auf einer Betonwand (Abb. 7), augenscheinlich durch Wasser ausgelöst, anzubeten. In den Flecken erkannten Katholiken ein Acheiropoieton, ein Bild nicht von Menschenhand, eine Marienerscheinung, das Gesicht der Maria. „Wir glauben, dass es ein Wunder ist «, sagte gemäß Frankfurter Allgemeine Zeitung die 42-jährige Elbia Tello: »Wir sind gläubig, und wir können ihr Gesicht sehen. «15 So wie bei den Street View-Maschinen fälschliche Gesichtserkennungen gesche-

15 http://www.spiegel.de/panorama/o,1518,352474,oo.html; http://www.faz.net/aktuell/gesellschaft/marienerscheinung-mutter-gottes-vom-kennedy-expressway-1232912.html. 
Abbildung 7 Gesichtsinterpretation beim Menschen: Verehrung eines natürlich erschienenen Marienbildnisses auf der Betonmauer einer Autobahnunterführung in Chicago

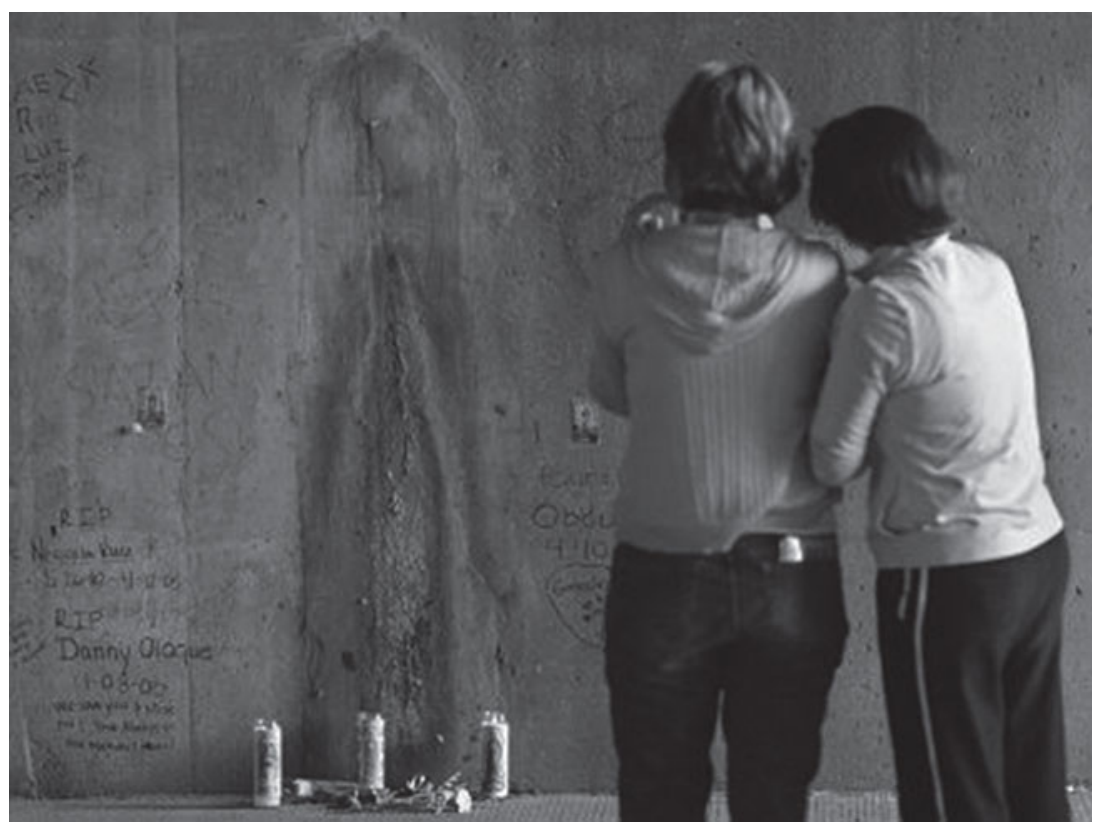

hen, kommen sie auch bei Menschen vor; sogar eine Krankheit, die Prosopagnosie, die pathologisch verminderte Fähigkeit zur Gesichtserkennung, wird diagnostiziert. Die Stabilität der Anwendung der Regeln zur Verarbeitung visueller Informationen, ob es sich nun um ein Gesicht handelt oder nicht, unterscheidet nicht die Interpretation visueller Information bei Maschinen und Menschen. Von den inneren Prozessen abstrahiert, lässt sich keine Differenz erkennen: Menschen und Maschinen interpretieren visuelle Informationen und erkennen in ihnen eine Gestalt. ${ }^{16}$ Mehr noch, Maschinen wie Menschen können ihre Auffassung

16 In Bezug auf die Interpretation von Gestalten, Gesichtern bleibt es müßig zu fragen, ließe sich mit Wittgenstein formulieren, was die Unterschiede der inneren Prozesse sind, entscheidend ist, dass das Vorstellungsbild, beispielsweise eines Würfels, mit einer Handlung korrekt in Verbindung gebracht wird, etwa der Aussage, »das ist ein Würfel «: unabhängig davon, auf welche Weise der Begriff, die Deutung des Konzepts > Würfel < auch verstanden wird; vgl. hierzu die Ausführungen in den philosophischen Untersuchungen (Wittgenstein 1984: 139-141). 
über das Vorliegen eines Gesichts gegenseitig übersetzen. Doch sowohl Menschen wie Maschinen gehen entlang dieser Regeln >fehl ‘, humane wie maschinelle Informationsverarbeitungsprozesse kennen >Grenzen $<$ der Interpretation. ${ }^{17}$ Freilich, die Menschen, die sich dafür interessieren, können die Interpretation der Maschinen nicht unbedingt verstehen: Das Innere des chinesischen Raums bleibt verborgen, und was darin geschieht, ist von fremden Entitäten auf fremde Weise erledigt, gleicht aber im Ergebnis der menschlichen Tätigkeit. Das ist möglicherweise der Grund der Rätsel, die mit den automatischen Bildern einhergehen. Doch weshalb geht von den nicht verstandenen Interpretationen auch eine Faszination aus?

\section{Ästhetik des Automatischen}

Hinsichtlich der Faszination, die sie auslösen, vergegenständlichen die automatischen Aufnahmen von Street View mehr eine Kontinuität denn einen Bruch. Street View führt ein Projekt der Automatisierung der Sichtbarmachung gesellschaftlicher Wirklichkeit konsequent weiter, treibt es zu neuen Dimensionen. Wie Bazin in seiner Ontologie des fotografischen Bildes über das Auftauchen der fotografischen Technik vermerkte, schiebt sich bereits mit dem Fotoapparat ein "Objekt zwischen das Ausgangsobjekt und seine Darstellung ", so dass schon damals, mit dem Entstehen der Fotografie ein »Bild von der uns umgebenden Welt automatisch « entsteht. Es »erhält die Bildkreation eine Autonomie ohne schöpferische Vermittlung des Menschen und nach einem strengen Determinismus " (Bazin 2004: 37). Schon das Fotografische an sich suspendiert die Autorschaft der Bilder. Der Begriff "Automat «, etymologisch eine "Maschine, die sich selbst bewegt «, bezeichnete schon früher einen Apparat, der »etwas von einem Lebewesen an sich hatte «, eine gewisse Autonomie besaß. ${ }^{18}$ Dahingehend lässt sich auch verstehen, dass Cartier-Bresson von seiner Leica wie von einem autonomen Wesen spricht, mit dem er eine besondere Partnerschaft eingegangen ist: »Ich hatte nun die Leica entdeckt: Sie wurde die Verlängerung meines Auges und verließ mich nicht mehr. Den ganzen Tag spazierte ich mit angespannten Sinnen und suchte in den Straßen nach Bildern, um das Leben auf frischer Tat zu greifen « (CartierBresson 1998: 12). Die Leica gilt in diesem Zitat als autonomes Wesen mit eigenem

17 Google selbst zelebriert dahingehend eine Schicksalsgemeinschaft von Menschen und Automaten: » Wie bei allem was Menschen oder Maschinen tun, gibt es einen geringen Anteil von Fällen, die nicht wie gewünscht bearbeitet werden « (i. e. unkenntlich gemacht werden, F. K.), vgl. http://maps.google.ch/help/maps/mystreetview/questions.html. 
Willen, aber auch die Bilder, die auf der Straße gefunden werden müssen, verfügen über eine eigenständige Existenz.

In der Suspension der Autorschaft in der Fotografie liegt ganz offenbar eine eigene Faszination, mit der bereits früh experimentiert und die möglichst weit getrieben wurde. Unschwer ist zu sehen, an welche immense Tradition der religiösen Magie nicht von Menschen gemachter Bilder sie anschließt: die Acheiropoieta, welche die humane Autorschaft negiert, und damit auf eine höhere Sphäre des nicht unmittelbar Kontrollierbaren verweist. ${ }^{19}$ Fotografien, so scheint es, vermögen in ihren Techniken ein ganz Anderes zu zeigen, eine bislang Unsichtbares, das in der Kamera zur Sichtbarkeit erhoben wird und zur Erkenntnis führt, "dass es eine ganz andere Natur ist, die zu der Kameras als die zum Auge spricht « (Benjamin 2003: 36). Diese Faszination des Automatischen durchzieht die Fotografie-Geschichte, und der Versuch, die Bildaufnahmen vollständig an eine technische Konfiguration zu delegieren, um neue Sichtbarkeiten des Realen zu evozieren, erscheint wie eine Konsequenz aus dieser Erfahrung. Die Kamera sieht, was der Mensch nicht gesehen hat, mehr noch, sie bestimmt selbst, was gesehen wird.

Eine exemplarisch ins technische gewandte Acheiropoieta-Erzeugung ist das frühe System der vollständig automatischen Bilderfassung, das Reverend John A. Scott im Jahre 1869 patentierte (vgl. Gethmann 1999: 17). Es beruhte auf einem raffinierten Mechanismus zur automatischen Fotografie, der in einen Fessel-Ballon montiert wurde. Die so erstellten Fotografien ersetzten für die Kirchgemeinde die Perspektive von oben angesichts des fehlenden Kirchturms. Gerüchteweise wurde das »selbständige, fliegende Auge « allerdings bereits auch zu Kontrollzwecken der Sichtung säumiger Kirchgänger eingesetzt (Gethmann 1999: 18). Beispielhaft kommt das Faszinosum der automatischen Fotografie auch in der Brieftaubenfotografie zum Ausdruck. Der Apotheker Julius Neubronner montierte anfangs des letzten Jahrhunderts eine Kamera unter einer Brieftaube (sie wurden damals gebraucht, um Rezepte zu überliefern), um automatische Bilder während des Fluges zu verfertigten (vgl. Brons 2006; siehe Abb. 8 oben). Die mit großem kommerziellen Aufwand angekündigten Fotografien appellierten zunächst an die üblichen Sehgewohnheiten, an postkartenartige Darstellungen der "Stadt mit ihren herrlichen Bauwerken. «Aber gemäß Brons gewannen die Fotografien das Publikum gerade dadurch, dass sie die gewohnten Perspektiven durchbrachen, Perspektiven zeigten, welche die Betrachter selbst nicht unmittelbar einnehmen konnten und die dadurch ebenso überraschten wie beeindruckten. Bereits hier zeigt sich die Faszination der Rätselhaftigkeit automatisch erzeugter Bilder, wenn der Apotheker Neubronner über das Entwickeln seiner Tauben-Bilder schreibt: »Die Bilder sind oft grundverschieden von denen, die wir zu sehen gewohnt sind, dass 
Abbildung 8 Brieftaubenfotografie des deutschen Militärs 1903 (oben). Bericht im Popular Science Monthly 1930 (unten)
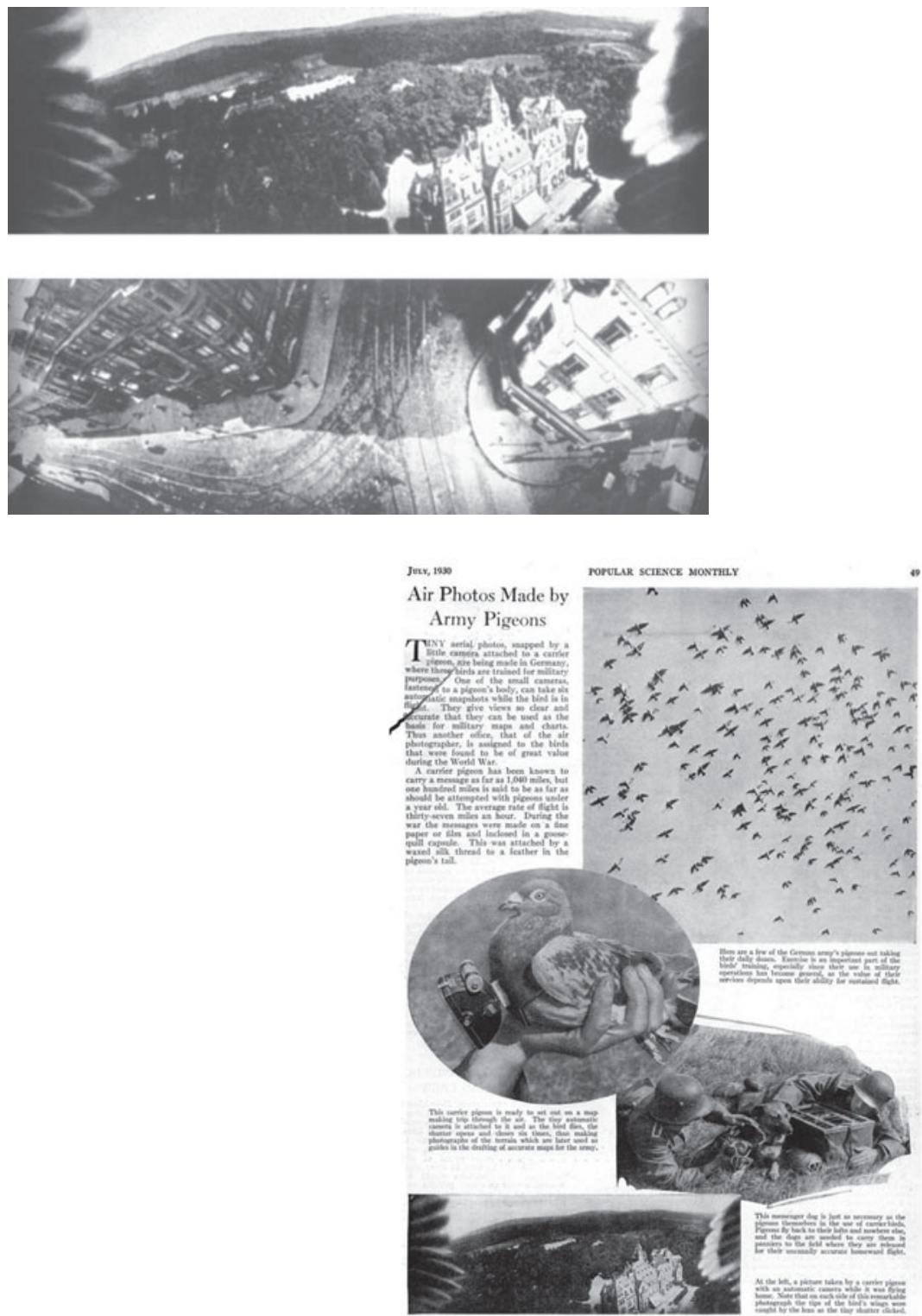
es große Mühe kostet, sich zurecht zu finden - Dieses übt einen ganz eigenartigen Reiz aus, den freilich nur wenige kennen. [...] Ich selbst muss zugeben, dass das einzige Taubenbild, welches zu entziffern mir bis jetzt nicht gelang, mich und meine Freunde lange Zeit in Aufregung versetzte (zitiert nach Brons 2006: 23).

Die automatischen Bilder aus der Luft, deren Möglichkeiten schnell auch das Militär erkannte (Abb. 8 unten), appellieren ganz offenbar an die Neugier darauf, dass die sichtbare Welt noch andere Perspektiven zulässt als die gewohnte des Alltagsmenschen, und diese Perspektiven wiederum schwierig zu dechiffrieren sind. Die Beobachtung der Welt aus der überraschenden Perspektive des nicht-menschlich Geschauten, erlebt mit dem Blick in die Natur, der Entwicklung der Kamerafalle und der Wildtierfotografie einen weiteren, vielleicht weniger diskutierten Sprung. Einer ihrer Pioniere war Frederick Walter Champion, ein britischer Förster, der zu Beginn des Jahrhunderts in den damaligen Vereinigten Provinzen Indiens arbeitete. ${ }^{20}$ Sein Anliegen war, verschwindende Tierarten vor die Kamera und ins Bewusstsein zu bringen, dafür das Gewehr, das in den Kriegen so viele Tote produziert hatte, durch die Kamera zu ersetzen und so seltenes Leben wieder sichtbar zu machen. Das Zufallselement des automatischen `Schießens` von Fotografien, die unvorhersehbaren Bilder, stipulierte die Spektakularisierung des Wilden, die sich auf andere Einrichtungen übertrug. So waren sogenannte Shooting-Bilder eine Zeitlang auf den Jahrmärkten populär: die Besucher schossen auf einen Auslösemechanismus und fanden sich automatisch, auf nicht vorhersehbare Weise, fotografiert (vgl. Chéroux 2010).

Wie die Jahrmarktfotografie, die Brieftauben- und Ballonfotografie nahelegen, erweisen sich nicht Fotografen oder Künstler als Pioniere der automatischen Fotografie und ihrer Ästhetik, sondern Amateure und Ingenieure, Pfarrer, Cowboys und Apotheker (vgl. Gethmann 1999: 17). Die Faszination der neuen maschinellen Sehweise und der rätselhaften neuen, nicht-menschlichen Perspektiven auf die Welt des Sichtbaren ergriff indes schnell auch die Künste. Exemplarisch ist hierfür die Leidenschaft der Surrealisten für die automatische Interpretation der Welt, die sich wiederum mit der Vorstellung des Unbewussten verband, das ebenfalls jenseits der verstehbaren Wahrnehmung- und Wahrnehmungstechniken arbeitet. Als im Paris der zoer Fotoautomaten zur Porträt-Erstellung auftauchten, wurden sie von den Surrealisten umgehend in Beschlag genommen und beinahe "zwanghaft « für ihre Experimente genutzt (vgl. Musée d'Élysée 2012). Die ästhetische Verbindung der automatischen Fotografie zum Konstrukt der écriture automatique erweist sich dabei als unmittelbar evident. Breton beispielsweise parallelisierte die Aufnahmetechnik der Kamera mit der écriture automatique, aufgrund

20 Vgl. zu seinen Erfahrungen, ebenso wie zu den technischen Erörterungen: Champion (2001) sowie Champion (1927). 
des Potentials beider Techniken, Unwahrgenommenes ins Bewusstsein zu heben. Das fotografische Verfahren kommt im "Prozess mechanischen Aufnehmens « einem "psychischen Automatismus " gleich (Krauss 1998: 112). Breton erklärt, dass für ihn das »automatische Schreiben, das Ende des 19. Jahrhunderts aufkam, eine regelrechte Photographie des Gedankens ist $"{ }^{21}$ eine Idee, die er in einem fotografisches Selbst-Porträt verwirklichte, das den Titel »L'écriture automatique « trägt und ihn vor einem Mikroskop zeigt, einem anderen Instrument zur Erhebung des »optisch Unbewussten «. In ähnlicher Weise faszinierte und beeinflusste die Geisterfotografie, die Erscheinung eines bislang Unsichtbaren über den automatischen Fotografie-Prozess, in ihrer Ästhetik die Künstler vom Surrealismus bis zur Gegenwart. ${ }^{22}$ Selbst der Fotoautomat bleibt nach den eingehenden Explorationen der Surrealisten bis in die heutige Zeit ein künstlerisches Untersuchungsobjekt. ${ }^{23}$

Von einer Ästhetik des Automatischen lässt sich dahingehend durchaus im ursprünglichen Wortsinn des Begriffs im Sinne einer aisthesis, einer Wahrnehmungsform, sprechen, die zugleich eine Interpretation realer Prozesse darstellt. Die automatische Bildgebung und Interpretation der sichtbaren Welt produziert andere Formen von Wahrnehmung, die zunächst von Ingenieuren und Amateuren hervorgebracht, im Feld der Kunst gleichsam >erforscht « werden. Die Magie anderer Sichtbarkeiten liegt dabei, unschwer erkenntlich, in der nicht vollumfänglichen Vorhersehbarkeit und Interpretierbarkeit dessen, was die Maschinen an Bildern, Perspektiven, Sichtbarkeiten produzieren: Solange, bis die neuen Perspektiven in den Bereich des gewohnten Sehens sedimentieren, wie es die überraschenden Bilder der Schnappschüsse taten, und deren Interpretation ebenso sautomatisch ' geschieht, wie sie erzeugt wurden. Die aufgrund automatischer Interpretationsprozesse aufgenommen Bilder faszinieren, so ließe sich vermuten, weil sie die ansonsten automatischen und unbewussten Interpretationsleistungen der Menschen über eine Irritation in Erinnerung rufen (vgl. Goodman/Elgin 1989: 154).

Folgerichtig wiederholt sich auch die künstlerische Auseinandersetzung mit den automatisch erzeugten Bilder und ihrem `Sinn`, ihre Heimholung in vertrautere Welten, bei den Street View-Bildern. Der eingangs erwähnte deutschamerikanische Fotograf und Künstler Michael Wolf sammelt "Zufallsfunde« aus

21 Zitiert nach Krauss (1998: 112).

22 Vgl. zum Einfluss der Geisterfotografie auf die Künste die Dokumentation des Fotomuseums Winterthur (Fischer et al. 1998).

23 Seine Fortsetzung findet dieser Prozess in der künstlerischen Auseinander mit automatischen Bildern in diversen Kunstprojekten, die sich mit Überwachungskameras beschäftigen; vgl. hierzu exemplarisch die Ausstellung "CTRL (Space) « in Karlsruhe sowie Pauleit (2005) und Kammerer (2008, Kap. VIII). 
Abbildung 9 Michael Wolf. street view paris. Ausstellung in Amsterdam 2010, präsentiert von foam fotografiemuseum amsterdam. Image \# 13

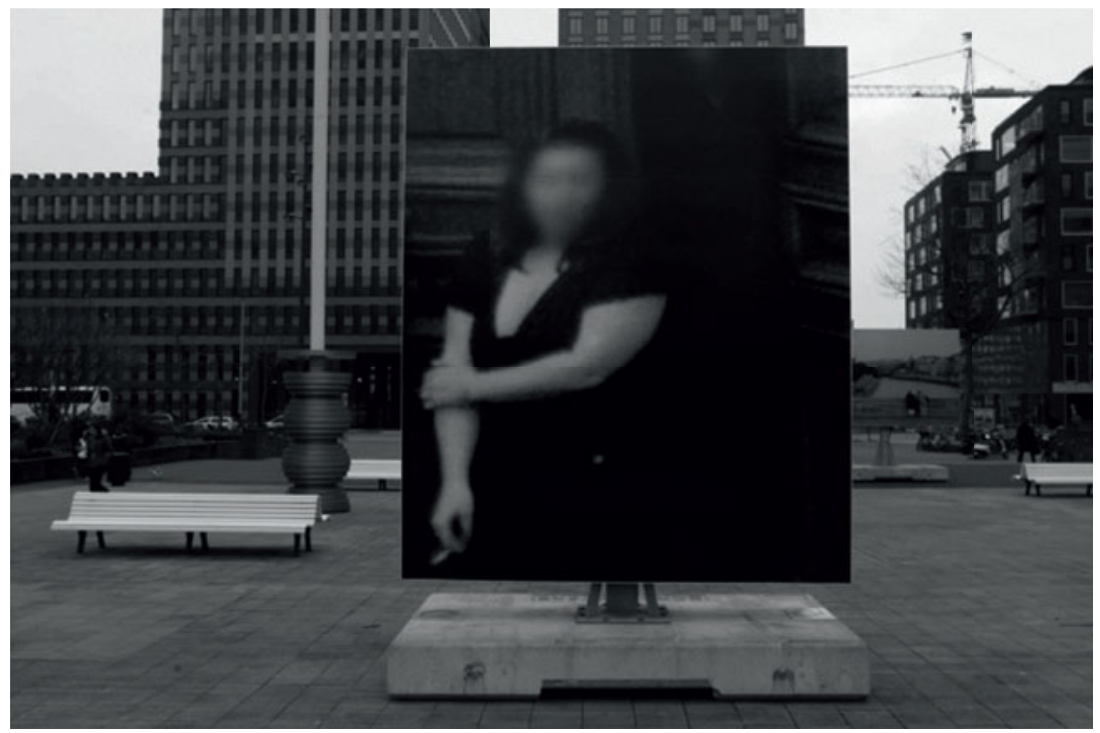

Google Street View, wie sie sich ihm auf der Bildschirmoberfläche zeigte, die er zu einem Kunstprojekt zusammenfasst, sie also in die Kunstwelt integriert und ihr als Invention und Intervention zur Interpretation übergibt. In besonderer Dichte kommt Wolfs Arbeit bei der obenstehenden Installation (Abb. 9) zum Ausdruck, gleichsam als Integration des schwerlich Interpretierbaren. Das in den öffentlichen Raum gestellte automatische Bild entstammt seiner Ausstellung paris street view in Amsterdam: das ausgestellte Objekt zeigt das Bild einer Stadt, Paris, die wie kaum eine andere als Objekt der Fotografie dient, in der alles schon fotografiert ist und die gleichzeitig eine Geburtsstätte der Fotografie überhaupt ist. Wolf holt die automatische Fotografie als Realobjekt in den Raum zurück und bannt sie zugleich in eine Anordnung, indem er sie als Autor, als Mensch fotografiert, im Raum positioniert und wieder fotografiert. Doch der fotografische Akt, durch einen Menschen betätigt, ist hierin nur noch ein schwindendes Element in einem Verweisungs- und Interpretationssystem, welche die Anordnung exemplarisch ins Bewusstsein rückt.

Freilich, etwas fällt auf: Das Street View-Bild ist übergroß, materiell dominant, und es ist gleichzeitig gerahmt: eingefasst, als müsste die Interpretation der Welt, 
die es vornimmt, irgendwie kontrolliert werden. ${ }^{24}$ Wolf stellt das Objekt hin, wie um zu zeigen, dass einmal mehr eine andere Wirklichkeit, die sich zwischen Bekanntes stellt und dieses neu verknüpft, nicht von Menschenhand stammt. Die entstandene Installation hat mit Googles visueller Welt gleich viel oder ebenso wenig zu tun, wie Googles Street View mit dem realen Leben der Stadt: ein Objekt, entfernt aus einer dynamischen Bilderwelt und montiert als stillgestandenes Stück Zeit in einem neuen Ort. Wie als Laborprobe einer fremden Lebensform in einem Reagenzglas steht dieses automatische Bild im öffentlichen Raum, seiner Umwelt ganz entrissen: zum Studium durch die Menschen selbst, die es geschaffen haben, die die erzeugte Welt aber ebenso wenig verstehen können, wie jene, die ihresgleichen in den Köpfen trägt. Angesichts dieses Bildes rätselt eine Spezies über sich selbst.

\section{Literatur}

Barthes, Roland (1989), Die helle Kammer. Bemerkung zur Photographie, Frankfurt am Main.

Bazin, André (2004, zuerst 1945), »Ontologie des photographischen Bildes «, in: ders. (Hg.), Was ist Film?, Berlin, S. 33-42.

Belting, Hans (2005), Das echte Bild. Bildfragen als Glaubensfragen, München.

Benjamin, Walter (2003), Das Kunstwerk im Zeitalter seiner technischen Reproduzierbarkeit. Drei Studien zur Kunstsoziologie, Frankfurt am Main.

Bredekamp, Horst (2010), Theorie des Bildakts. Frankfurter Adorno-Vorlesungen 2007, Berlin.

Brons, Franziska (2006), »Bilder im Fluge. Julius Neubronns Brieftaubenfotografie «, in: Fotogeschichte. Beiträge zur Geschichte und Ästhetik der Fotografie 26, S. 17-36.

Cartier-Bresson, Henri (1998), Auf der Suche nach dem rechten Augenblick. Aufsätze zur Photographie und Erinnerungen an Freunde Berlin, München.

Champion, Frederick Walter (1927), With a camera in tiger-land. With plates and a bibliography, London.

Champion, Frederick Walter (2001), Preserving Wildlife in the United Provinces, New Delhi. Chéroux, Clément (2010), Shoot!: la photographie existentielle, Berlin.

Crary, Jonathan (1996), Techniken des Betrachters. Sehen und Moderne im 19. Jahrhundert, Dresden.

Doesinger, Stephan (2012), Der veröffentlichte Raum, Berlin.

Dubois, Philippe (1998), Der fotografische Akt. Versuch über ein theoretisches Dispositiv, hgg. und mit einem Vorwort von Herta Wolf, Amsterdam/Dresden.

Eco, Umberto (1995), Die Grenzen der Interpretation, München.

24 Die Bedeutung des Rahmens hinsichtlich der Konstitution eines Kunstwerks hat Simmel (1995) ja ausführlich diskutiert. 
Fischer, Andreas/Aigner, Carl/Stahel, Urs (1998), Im Reich der Phantome. Fotografie des Unsichtbaren, Ostfildern-Ruit.

Flusser, Vilém (1997), Für eine Philosophie der Fotografie, Göttingen.

Gadamer, Hans-Georg (1986), »Text und Interpretation «, in: Hans-Georg Gadamer (Hg.), Hermeneutik II. Wahrheit und Methode, Tübingen, S. 330-360.

Gethmann, Daniel (1999), »Unbemannte Kamera. Zur Geschichte der automatischen Kamera aus der Luft «, in: Fotogeschichte. Beiträge zur Geschichte und Ästhetik der Fotografie 19, S. 17-27.

Gombrich, Ernst H. (1984), »Kriterien der Wirklichkeitstreue: Der fixierte und der schweifende Blick«, in: ders., Bild und Auge: neue Studien zur Psychologie der bildlichen Darstellung, Stuttgart, S. 240-273.

Goodman, Nelson/Elgin, Catherine Z. (1989), Revisionen. Philosophie und andere Künste und Wissenschaften, Frankfurt am Main.

Günzel, Stephan (2011), »>In Real Life « Zum Verhältnis von Computerspiel und Alltag «, in: Marotzki, Winfried/Iske, Stefan/Fromme, Johannes (Hg.), Medialität und Realität: Zur konstitutiven Kraft der Medien, Wiesbaden, S. 159-176.

Kammerer, Dietmar (2008), Bilder der Überwachung, Frankfurt am Main.

Krauss, Rosalind E. (1998), Das Photographische. Eine Theorie der Abstände, München.

Krämer, Sybille (2008), Medium, Bote, Übertragung. Kleine Metaphysik der Medialität, Frankfurt am Main.

Lacan, Jacques (1991), »Das symbolische Universum «, in: ders., Das Seminar von Jacques Lacan Buch II (1954-1955), Weinheim/Berlin, S. 39-54.

Manovich, Lev (2001), The Language of New Media, Cambridge/Massachusetts/London.

Mitchell, W. J.T (2008), Das Leben der Bilder. Eine Theorie der visuellen Kultur, München.

Mittelstrass, Jürgen (1995), Enzyklopädie Philosophie und Wissenschaftstheorie. Bd. 2: H-O, Stuttgart/Weimar.

Musée d'Élysée (2012), Derrière le rideau. L'Esthétique Photomaton des surréalistes à Cindy Sherman en passant par Andy Warhol, Lausanne.

Pauleit, Winfried (2005), »Photographesomenon. Videoüberwachung und bildende Kunst «, in: Hempel, Leon/Metelmann, Jörg (Hg.), Bild - Raum - Kontrolle. Videoüberwachung als Zeichen gesellschaftlichen Wandels, Frankfurt am Main, S. 73-90.

Perrottet, Oliver (2011), Taxi, Zürich.

Searle, John R. (2009), »Chinese room argument «, in: Scholarpedia 4:310o http://www.scholarpedia.org/article/Chinese_room_argument.

Searle, John R. (2008), »Twenty-one years in the Chinese Room «, in: Philosophy in a New Century: Selected Essays, Cambridge.

Serres, Michel/Auffray, Charles (2001), Thesaurus der exakten Wissenschaften, Frankfurt am Main.

Scott, Clive (2007), Street Photography. From Atget to Cartier-Bresson, London.

Simmel, Georg (1995, zuerst 1902), »Der Bildrahmen. Ein ästhetischer Versuch «, in: ders., Aufsätze und Abhandlungen 1901-1908, Frankfurt am Main, S. 101-108.

Stegmüller, Wolfgang (1989), Hauptströmungen der Gegenwartsphilosophie: eine kritische Einführung. Band 1, Stuttgart.

Stiegler, Bernd (2009), Montagen des Realen: Photographie als Reflexionsmedium und Kulturtechnik, München. 
Stock, Alex (1996), Poetische Dogmatik. Christologie. Schrift und Gesicht, Ort, Paderborn. Virilio, Paul (1989), Die Sehmaschine, Berlin.

Wittgenstein, Ludwig (1984), Tractatus logico-philosophicus. Tagebücher 1914-1916. Philosophische Untersuchungen. Werkausgabe Band 1, Frankfurt am Main.

\section{Abbildungen}

Abb. 1: http://www.photomichaelwolf.com/paris_street_view/; Abb. 2: http://maps.google. ch/intl/de_ch/help/maps/streetview/learn/cars-trikes-and-more.html; Abb. 3: http://www. photomichaelwolf.com/street_view_unfortunate_events; Abb. 4, links: Perrottet, Oliver. Taxi. Zürich: Ed. Frey, 2011, auch online: http://www.zeit.de/reisen/2012-02/fs-taxi-oliverperrottet-2/seite-2; Abb. 4, rechts: Google Streetview. Selbsterstellter Screenshot; Abb. 5, links: Gombrich, Ernst H. (1984), »Kriterien der Wirklichkeitstreue: Der fixierte und der schweifende Blick«, in: Bild und Auge: Neue Studien zur Psychologie der bildlichen Darstellung. Stuttgart, Abbildung 233 (Ausschnitt); Abb. 5, rechts: http://www.photomichaelwolf.com/paris_street_view/; Abb. 6: Google Street View, selbsterstellte Screenshots; Abb. 7:http://www.faz.net/aktuell/gesellschaft/marienerscheinung-mutter-gottes-vomkennedy-expressway-1232912.html; Abb. 8, oben: http://www.geog.ucsb.edu/ jeff/115a/history/pigeonremotesensing.html; Abb. 8, unten: Popular Science Monthly, July, 1930, S. 49; Abb 9: http://www.photomichaelwolf.com/street_view_installation_photos/Paris. 